\title{
Las primeras mujeres juristas de Cataluña. Evolución del número de mujeres estudiantes de Derecho en Cataluña y colegiadas en Barcelona entre 1910 y 1968
}

The first women jurists in Catalonia. Evolution of the number of Law women students in Catalonia and registered lawyers in Barcelona between 1910 and 1968

Patrícia Soler i Penya

Graduada en Derecho

Facultad de Derecho, Universidad de Barcelona.

E-mail: solerpenyapatricia@gmail.com

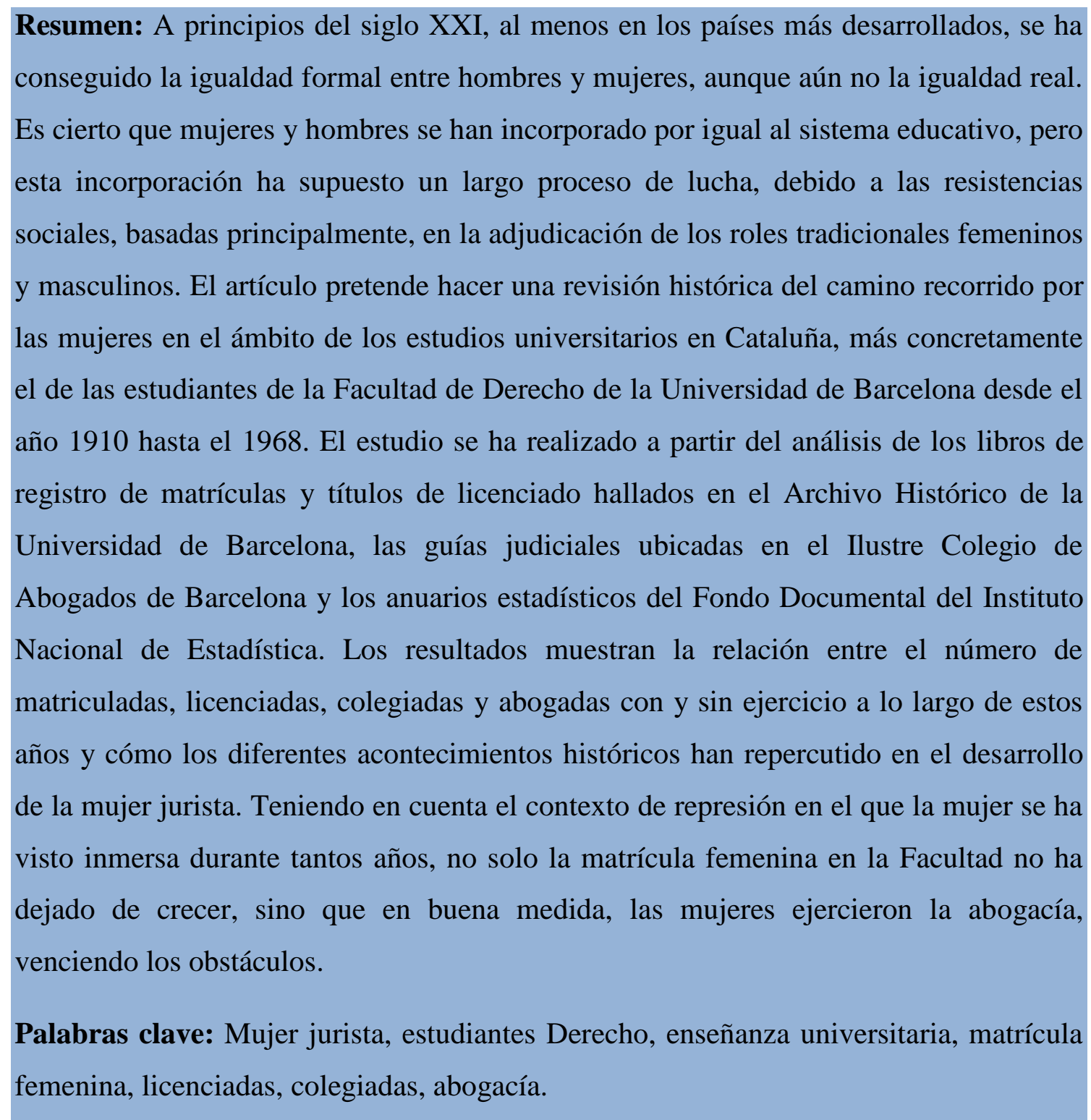


Abstract: At the beginning of the 21st century, at least in the more developed countries, formal equality between men and women has been achieved, although not yet real equality. It is true that women and men have joined the educational system equally but this incorporation has been a long process of struggle, due to social resistance, based mainly on the adjudication of traditional feminine and masculine roles. The work aims to make a historical review of the path taken by women in the field of university education in Catalonia, more specifically that of the students of the Faculty of Law of the University of Barcelona from 1910 to 1968. The study is based on the analysis of the registration books and degree titles found in the Historical Archive of the University of Barcelona, the judicial guides located in the Barcelona bar association and the statistical yearbooks of the national institute documentary fund of statistics. The results show the relationship between the number of enrolled, graduated, registered lawyers and lawyers with and without exercise throughout these years and how the different historical events have had an impact on the development of women lawyers. Taking into account the context of repression in which women have been immersed for so many years, not only has female enrollment in the Faculty not ceased to grow, but to a large extent, women practiced law, overcoming obstacles.

Keywords: Female lawyer, law students, university education, female tuition, graduates, registered women lawyers, law.

\section{Objeto y delimitación del estudio}

Hoy en día sabemos que la primera mujer que se licenció en Derecho en Cataluña, en la Universidad de Barcelona (UB), y la primera que se inscribió en el Colegio de Abogados de Barcelona, en 1927, fue María Soteras Mauri (Barcelona, 4 de diciembre de 1905 - Ciudad de México, 9 de marzo de 1976) (Memòria, 2015). Sin embargo, más allá de este dato, es muy poca la información que se encuentra respecto de la mujer jurista en Cataluña durante la primera mitad del siglo XX.

Ahora presentamos los resultados de un estudio que contempla la evolución de la mujer en el mundo de la abogacía en Cataluña desde 1910 hasta $1968 .^{1}$ El periodo que comprende este análisis (1910 - 1968) responde a dos razones:

\footnotetext{
${ }^{1}$ El origen de este texto es un Trabajo Final de Grado (TFG) con el título "Les primeres dones juristes de Catalunya. Evolució del nombre de dones estudiants de Dret i col-legiades a Catalunya (1910 a 1968)" (Las primeras mujeres juristas de Cataluña. Evolución del número de mujeres estudiantes de Derecho y colegiadas en Cataluña (1910 a 1968), presentado en la Facultad de Derecho de la UB en el curso 201819 y tutorizado por el profesor Max Turull Rubinat, del Departamento de Historia del Derecho, Derecho
} 
En primer lugar, Itatí $(2006,12)$ distingue dos períodos en relación con el acceso de las mujeres a la educación universitaria: en el primero, algunas pocas mujeres accedieron de manera "excepcional" o disfrazadas de hombres; en el segundo, el que llamamos proceso sistemático que se inicia en 1910, se produce el acceso de la mujer "como género". Es con la Real Orden de 8 de marzo de 1910 dictada por el Ministro de Instrucción pública que se autoriza a las mujeres a acceder a la educación superior en España sin necesidad de un permiso especial. Es por este motivo que el presente estudio tiene como punto de partida el año 1910.

En segundo lugar, la UB fue la única universidad de Cataluña y las Islas Baleares existente hasta el año 1968, cuando se inició un proceso (que duró hasta la década de los 90) que originó el resto de las universidades catalanas y la Universidad de las Islas Baleares, por lo que el presente estudio finaliza en el año 1968 (Andreu, López, Vernet, 2013, 132).

Los objetivos que se desean alcanzar con esta contribución son consolidar y depurar los datos de las fuentes de archivo de cara a un primer conocimiento del tema para facilitar futuros trabajos; conocer quienes fueron las primeras mujeres que se matricularon en la Facultad de Derecho de la UB; analizar la presencia de mujeres en la Facultad (1910 a 1968); estudiar la relación entre matriculadas, licenciadas, colegiadas y abogadas con y sin ejercicio (1910 - 1968); interpretar los datos obtenidos a partir de las fuentes primarias y explicar el porqué de la evolución que siguen y, finalmente, visibilizar, tomar conciencia y revisar la posición de la mujer jurista desde sus inicios.

\section{Marco teórico}

El siglo XX trajo consigo un gran progreso para las mujeres, abriendo las puertas a un debate sobre su papel social, económico y político. La lucha por alcanzar la igualdad política, jurídica y económica fue uno de los grandes fenómenos del siglo pasado.

Romano y Derecho Eclesiástico del Estado. A lo largo del artículo aparecen los nombres y apellidos de algunas de las pioneras. Si bien se trata de datos personales y, en consecuencia, son objeto de la normativa de protección de datos, ésta no es aplicable cuando la persona afectada sea difunta (Considerando 27 del Reglamento (UE) 2016/679 del Parlamento Europeo y del Consejo, de 27 de abril de 2016, relativo a la protección de las personas físicas en lo que respecta al tratamiento de datos personales y a la libre circulación de estos datos y por el que se deroga la Directiva 95/46/CE (Reglamento General de Protección de Datos), y el art. 2.2 b Ley Orgánica 3/2018, de 5 de diciembre, de Protección de Datos Personales y Garantía de los Derechos Digitales). Teniendo en cuenta la fecha en la que se licenciaron, que como muy pronto se licenciaron con 23 años, y que la esperanza de vida para las mujeres nacidas en el año 1975 es de 76,3 años según el INE, podemos llegar a la conclusión de que se trata de personas difuntas y que, por lo tanto, sus nombres y apellidos pueden ser publicados sin contravenir la normativa de protección de datos. 
Foraster $(2015,8)$ destaca que "después de la Revolución Francesa se elaboró un discurso político, derivado de la Ilustración, de contenido igualitario, el cual se dirigía a garantizar los derechos políticos a todos los ciudadanos. Sin embargo, la práctica política no lo garantizaba, especialmente cuando intervenía la desigualdad de sexos, ya que, por ejemplo, los partidos políticos no admitían a las mujeres dentro de sus filas. Este marco teórico fabricado desde el poder que favorecía la exclusión de la mujer del espacio público de la política, perduró hasta las primeras décadas del siglo XX, también en España, donde el discurso ideológico-cultural dominante, muy conservador, favorecía esta realidad diferencial de sexos".

La doctrina (Gómez, 2019) afirma que es en este momento, a principios del siglo XX, cuando empezó el feminismo en Cataluña, un feminismo de carácter cultural y social, cuestionando la exclusión de las mujeres del mundo de la cultura y la subordinación femenina. Se focalizó en la promoción de los derechos de las mujeres en los ámbitos educativos, culturales y laborales, porque las características del desarrollo político español durante la Restauración borbónica no fueron favorables para la emergencia de un feminismo liberal de carácter político.

Según Moreno (2010), la conciencia social de la mujer percibió un giro con la Primera Guerra Mundial. Mientras los hombres luchaban en el frente durante el conflicto, el mantenimiento de la economía productiva fue asumido por la mujer, lo que suponía para ésta un nuevo lugar en la sociedad. En Alemania y en Gran Bretaña, la mano de obra industrial femenina constituía el 35\% a finales del conflicto bélico. Pero, además, las mujeres se empezaron a incorporar a la actividad laboral en las oficinas y en las profesiones liberales. De este modo, uno de los grandes temas de la época de entreguerras sería la emancipación femenina. Así, en 1918, Gran Bretaña concedió el derecho a voto de las mujeres de más de 30 años y a todos los hombres mayores de 21 años y, en 1928, se igualó la edad de voto y, por primera vez, poder entrar mujeres en el Parlamento (Moreno, 2010).

Además de la propia experiencia de exclusión social y de desigualdad, la recepción del discurso ilustrado en España durante la II República, impulsó a muchas mujeres republicanas y obreras a reclamar su identidad de sujeto político en igualdad de derechos con el sujeto masculino. También quisieron hacer visibles sus propias reivindicaciones como, por ejemplo, el acceso al espacio público de la política. Aun siendo diversas las posturas del colectivo femenino respecto a su integración en la 
política, se hizo patente un incremento de la salvaguarda de derechos políticos, centrados especialmente en la conquista del derecho al voto femenino. La aprobación del sufragio en las Cortes Constituyentes de la II República (1931) fue el resultado de este proceso. En países como Francia e Italia no se consiguió el derecho al voto femenino hasta después de la Segunda Guerra Mundial (Foraster, 2015, 8).

Gracias al contexto histórico transicional de la II República se cuestionaron relaciones de género a todos los niveles y se aprobaron leyes como la ley del divorcio, de la coeducación, del derecho de voto femenino, del matrimonio civil o la progresiva legislación laboral igualitaria. Por otro lado, y aunque actuaron mayoritariamente durante la Guerra Civil (1936-39), habían nacido durante la II República gran parte de las primeras organizaciones políticas femeninas (Foraster, 2015, 9).

Iniciada la guerra civil de 1936-39, Federica Montseny, la ministra de sanidad del gobierno de Largo Caballero, apoyó el decreto sobre el aborto, aprobado en diciembre de 1936 por la Generalitat de Cataluña. El transcurrir de la guerra requería una participación real de las mujeres en las tareas de servicios públicos, de industria de guerra, etc. Adscrito a la consejería de trabajo, se creó, en julio de 1937, el Instituto de Adaptación Profesional de la Mujer, el cual, en marzo de 1938, había congregado a 5.000 mujeres para el trabajo. Convocado por mujeres de la la CNT, UGT, el Partido Sindicalista, la ACR, el PSUC, el ERC, el Partido Federal Ibérico y el Estado Catalán, se celebró en el Palau de la Música de Barcelona, el Primer Congreso Nacional de la Mujer, en noviembre de 1937; asistieron 800 delegadas, y se decidió la creación de la Unión de Mujeres de Cataluña, que luego organizó la Jornada Internacional de la Mujer en Barcelona (Ackelsberg, 2017).

Toda reivindicación feminista se vio paralizada por la posguerra y el régimen franquista; fue la "Sección Femenina de la FET y de las JONS" la única organización femenina autorizada. No se volvió a promover el interés por este tema hasta los años sesenta y una de las figuras más destacadas de este despertar fue Maria Aurèlia Capmany (Foguet, 2018).

En la gran mayoría de países, incluidos los Estados europeos, las mujeres casadas no podían disponer de sus bienes, tener la custodia de los hijos, firmar contratos ni disponer libremente de sus personas hasta después de la Primera Guerra Mundial. La igualdad jurídica se estableció en Gran Bretaña en 1925, en 1938 en Francia, y en los años cincuenta en Alemania. En cambio, en España, las mujeres casadas no pudieron 
adquirir el carné de conducir o tener pasaporte sin la aprobación del esposo hasta el fin de la dictadura franquista (Moreno, 2010).

\section{Metodología}

En cuanto al estudio de la matrícula femenina, los anuarios estadísticos del Fondo Documental del Instituto Nacional de Estadística (INE) (1912-68) y los libros de registro de matrículas en la Facultad de Derecho de la UB han sido las fuentes principales utilizadas.

Con respecto al análisis del número de licenciadas, las fuentes primarias empleadas han sido los libros de registro de los títulos de licenciado en Derecho por la UB hallados en la Facultad (1910-1947 y 1957-1969) y la relación de títulos de licenciado pagados de las diferentes Facultades de la UB ubicada en el Archivo Histórico de la UB (19471957).

Finalmente, por lo que se refiere al estudio del número de abogadas colegiadas y su ejercicio profesional, las fuentes consultadas han sido las Guías Judiciales del Ilustre Colegio de Abogados de Barcelona (ICAB) de 1927-1968. 


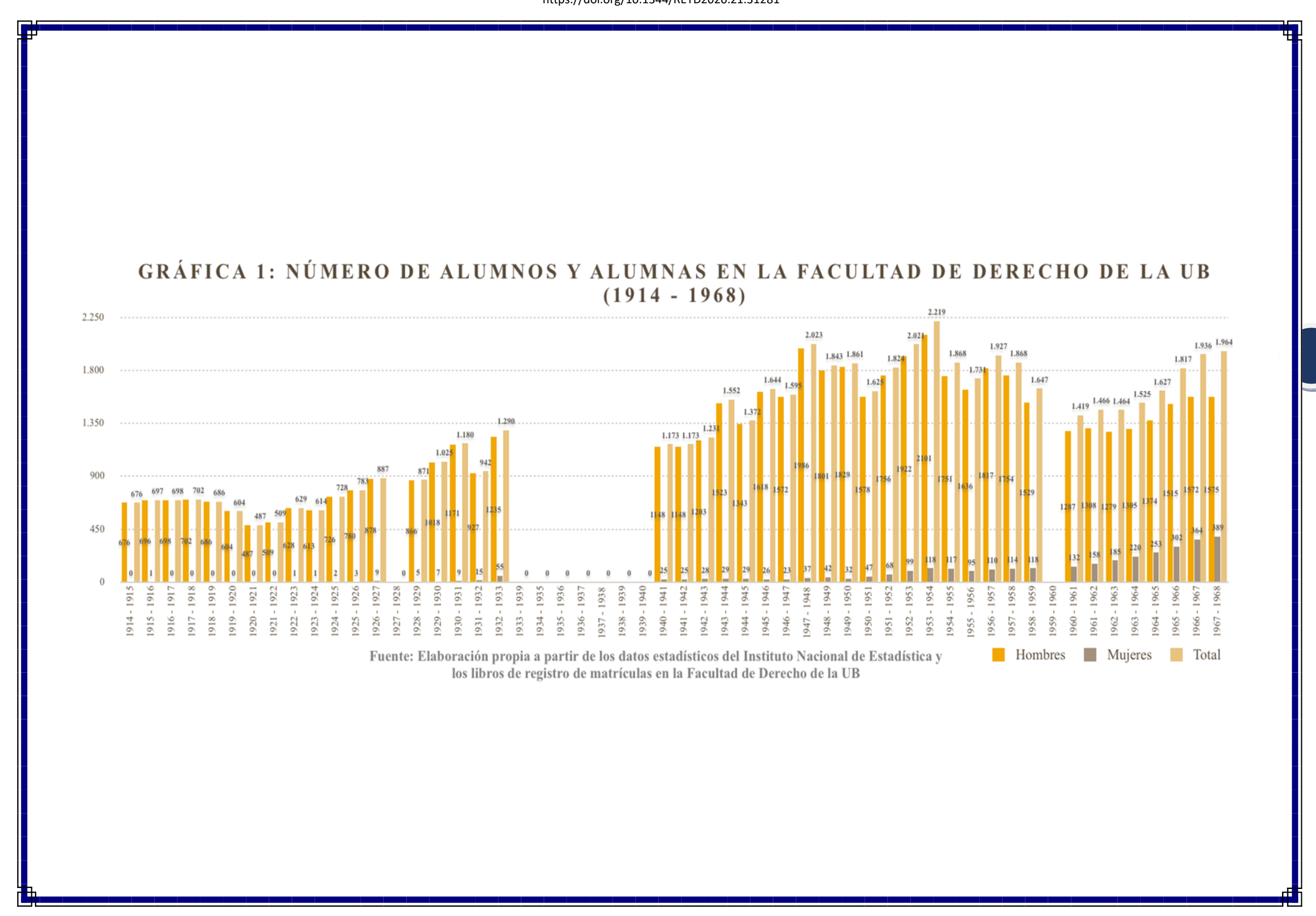


Patrícia Soler i Penya. Las primeras mujeres juristas de Cataluña. Evolución del número de mujeres estudiantes de Derecho en Cataluña y colegiadas en Barcelona entre 1910 y 1968

TABLA 1: NÚMERO DE MUJERES QUE INICIAN DERECHO Y NÚMERO DE LICENCIADAS (1915-1932)

\begin{tabular}{|c|c|c|}
\hline Curso & $\begin{array}{l}\text { Número de mujeres matriculadas que } \\
\text { inician la carrera }\end{array}$ & De las que la inician, cuantas se licencían? \\
\hline $1915-1916$ & 1 & 0 \\
\hline $1916-1917$ & 0 & 0 \\
\hline 1917 - 1918 & 0 & 0 \\
\hline $1918-1919$ & 0 & 0 \\
\hline $1919-1920$ & 0 & 0 \\
\hline $1920-1921$ & 0 & 0 \\
\hline $1921-1922$ & 0 & 0 \\
\hline $1922-1923$ & 1 & 1 \\
\hline $1923-1924$ & 0 & 0 \\
\hline $1924-1925$ & 1 & 0 \\
\hline $1925-1926$ & 2 & 1 \\
\hline $1926-1927$ & 4 & 2 \\
\hline \multicolumn{3}{|l|}{$1927-1928$} \\
\hline \multicolumn{3}{|l|}{$1928-1929$} \\
\hline $1929-1930$ & 4 & 0 \\
\hline $1930-1931$ & 7 & 3 \\
\hline $1931-1932$ & 8 & 3 \\
\hline $1932-1933$ & 10 & 2 \\
\hline
\end{tabular}

Fuente: Elaboración propia a partir de los libros de registro de matrículas y de los libros de registro de títulos de licenciado en Derecho por la UB 
Patrícia Soler i Penya. Las primeras mujeres juristas de Cataluña. Evolución del número de mujeres estudiantes de Derecho en Cataluña y colegiadas en Barcelona entre 1910 y 1968

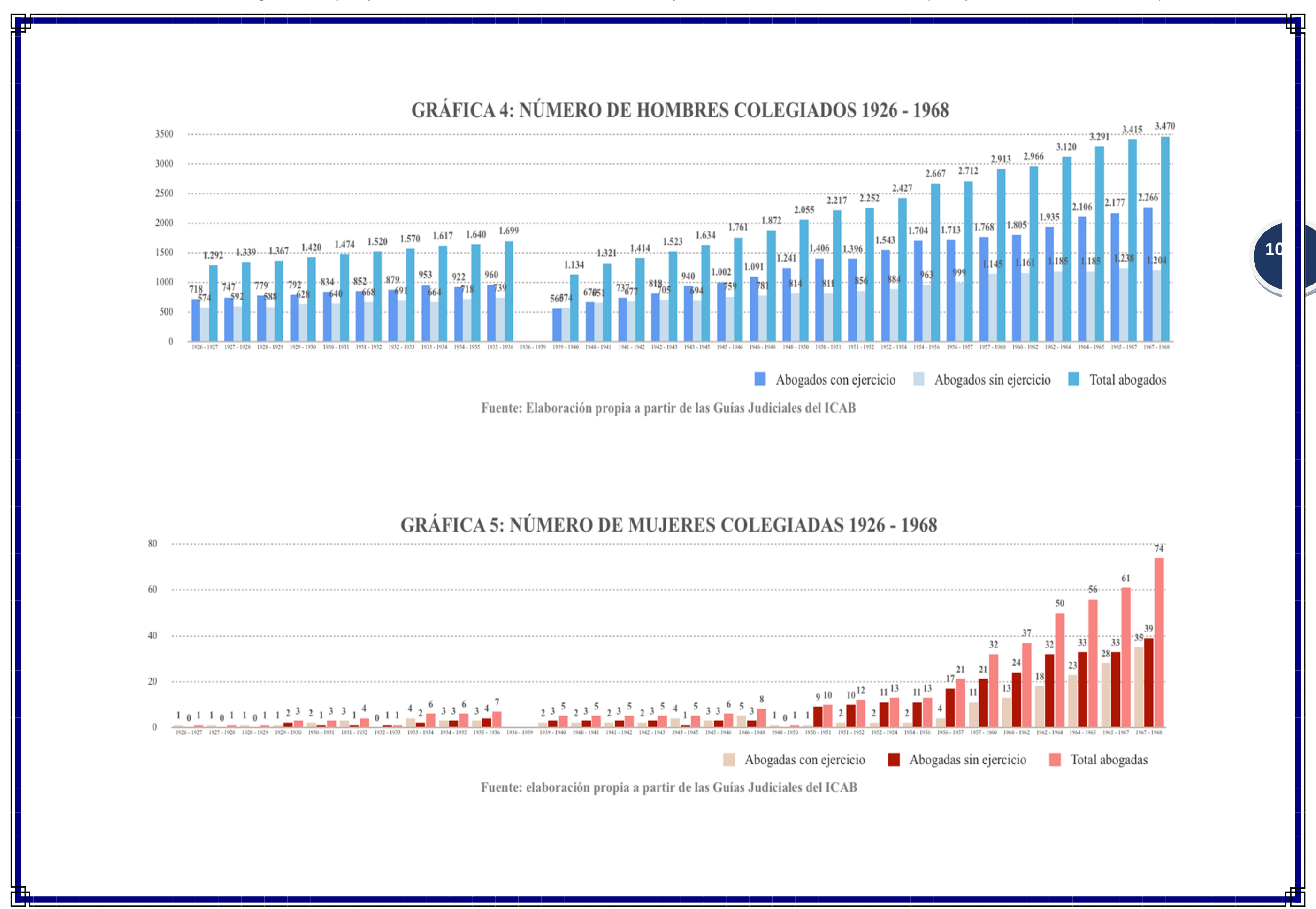




\section{Resultados}

\subsection{Las estudiantes de Derecho en Cataluña}

Según Del Amo $(2009,15)$, a pesar de haberse regulado la admisión de las mujeres a la universidad en las mismas condiciones que los hombres en 1910, la presencia de la mujer en la facultad es ínfima durante las primeras décadas del siglo. Pese a las nuevas disposiciones legales, había pocas mujeres matriculadas en el bachillerato y en consecuencia pocas tenían el título de bachillerato imprescindible para cursar estos estudios. Además, estas pioneras convivían con la actitud hostil de profesores y compañeros, la oposición familiar, dificultades sociales para el ejercicio profesional de los conocimientos adquiridos, etc.

La primera mujer que se matriculó en la Facultad lo hizo en 1916 y fue la única del curso 1915-16. Se trataba de María del Amparo Roig García ${ }^{2}$ y no será hasta el 1922 que se matriculará otra mujer, Maria Soteras Mauri (reg. matrícula 1914-1920). A partir de este momento el número de mujeres va aumentando progresivamente durante los años siguientes, no en gran medida ni en la misma proporción que los hombres, pero, tal y como observamos en la Gráfica 1, en 1930 son 9 las mujeres matriculadas y representan el $0,8 \%$ de la totalidad del alumnado.

En cuanto al periodo de la II República, el vacío de información entre los años 1933 y 1939 no nos permite determinar la evolución de la presencia femenina en la Facultad durante este periodo. No obstante, sí observamos que en 1931, son 15 las mujeres matriculadas, representando el 1,6\% de la totalidad del alumnado (INE 1932-33), y aumentan a 55 en 1932, representando el 4,3\% (INE 1934). Pues según García (2010, 362), la II República es un periodo histórico especialmente fructífero para la situación de las mujeres en toda España, lo que provocó que su presencia en el mundo educativo y laboral se incrementara de manera significativa. Durante la II República culmina el incremento cuantitativo y cualitativo de universitarias que se había iniciado en los años veinte representando en el curso 1935-36 el 9\% del alumnado, una cifra que debe compararse con el 0,1\% en el momento en que fue aprobada la Real Orden de 1910.

\footnotetext{
${ }^{2}$ No sabemos si María del Amparo Roig García terminó la carrera de Derecho en otra universidad, pero podemos afirmar que no lo hizo en la Facultad de Derecho de la UB. De acuerdo con la documentación consultada para la realización del presente artículo, venía de traslado de Madrid y solo se matriculó de la asignatura de Derecho Administrativo del tercer curso, quedándole por cursar las asignaturas del cuarto y quinto curso, así como las restantes del tercero.
} 
Las mujeres se concentraban fundamentalmente en los estudios de Farmacia, Filosofía y Letras, Ciencias y Medicina. Pero, sin duda, el cambio más relevante en estos años consiste en que, por primera vez, se acepta con naturalidad que la titulación universitaria de las mujeres puede implicar un ejercicio profesional. Aparecen las primeras profesoras de bachillerato, inspectoras de educación, profesoras contratadas por la Universidad, etc. (García, 2010, 362).

Solo durante el primer año de la guerra se interrumpió la docencia, especialmente para los alumnos que luchaban en el frente, y en septiembre de 1936 se aplazaron sin fecha los exámenes de ingreso y la matrícula universitaria. Durante aquel curso de 1936-37 la actividad se centró en la protección del patrimonio histórico y bibliográfico, el acondicionamiento de edificios y la investigación en los seminarios. El gobierno de la Generalitat decidió normalizar la vida académica al curso siguiente, el de 1937-38, cuando se reanudaron las clases y el régimen de autonomía en la Universidad. El 18 de marzo de 1938, a consecuencia del bombardeo de la aviación italiana, varios edificios en la Gran Vía quedaron derrumbados y el edificio de la Universidad sufrió graves desperfectos en los tejados, techos y ventanas. Evidentemente, se tuvieron que suspender las clases. Pasado el difícil momento de las operaciones militares del invierno y la primavera de 1938, el curso 1938-39 se intentó preparar con normalidad. (Fullola, 2014, 38-44). Así, dadas las circunstancias especiales marcadas por la guerra, la documentación relativa a los años 1936-39 es muy incompleta e incluso, en algunos casos, inexistente, por lo que el gráfico no muestra ningún dato al respecto.

Una vez terminada la Guerra Civil, se produce la reincorporación de algunas mujeres en la Facultad. El número de alumnos, tanto de hombres como de mujeres, baja de manera importante. Pasamos de un total de 1.290 en el curso 1932-33, a un total de 1.173 en el curso 1940-41. Mientras el 1932-33 hay 55 alumnas matriculadas que representan el 4,3\% del total, el 1940-41 hay 25 y representan el 2,1\% (INE 1943). No será hasta el curso 1953-54 que el número de mujeres matriculadas se acercará de nuevo al 5\% del alumnado.

¿A qué se debe este descenso? Flecha $(1989,79)$ destaca que "la política de las autoridades educativas franquistas, en el marco de la estructura ideológica sobre la que se va constituyendo el nuevo Estado, fue respondiendo a las exigencias de socialización derivadas del modelo propuesto: una mujer llamada a desarrollar un doble papel de esposa y madre". 
Del curso 1940-41 hasta el 1951-52, como se muestra en la Gráfica 1, el número de mujeres se mantiene más o menos estable, en el sentido de que el porcentaje que representan respecto al total del alumnado va variando, pero siempre se mantiene entre el 2 y el 3\%. Es a partir del curso 1952-53 que aumenta en mayor medida y, como hemos dicho antes, es en el curso 1953-54 cuando, transcurridos casi 20 años, se recupera la cifra de la II República con un 4,9\%. A partir de este momento, el porcentaje que representarán las mujeres respecto a la totalidad del alumnado ya no dejará de crecer.

Tal y como se puede ver en la Gráfica 1, en el curso 1953-54 son 118 las mujeres matriculadas en la Facultad de Derecho de la UB y el mismo número se repite en el 1958-59. Entre estos dos cursos, el de 1955-56 es el que contiene el número más bajo de matriculadas, concretamente 95. Así, el número de mujeres matriculadas durante este periodo se mantiene más o menos estable.

Según algunos autores (García, 2010, 362), “a partir de los años 60, se produjo en España el llamado "milagro español" o época del desarrollismo, esto es, una etapa que desde el punto de vista económico significó un nuevo escenario productivo que conllevó el inicio de una incipiente apertura sociopolítica que implicó la progresiva transformación de la realidad española, en la que el papel de las mujeres era una de sus piezas. De este modo y aunque todavía tienen que pasar algunos años para que las mujeres obtengan algunos derechos básicos, su situación social empieza a ser percibida de manera diferente a lo que hasta entonces había sido considerada. Así, de manera progresiva, las mujeres van alcanzando derechos básicos arrebatados por el régimen franquista y accediendo a espacios en otras épocas vetados".

Es precisamente a partir de este momento cuando el número de mujeres comienza a ser significativo. Durante el periodo que comprende los cursos 1960-61 hasta el 1967-68, el número de hombres matriculados en la Facultad aumenta en gran medida, pero lo hace de manera más relevante y constante el de mujeres. Así, en el curso 1960-61, son 132 mujeres matriculadas representando el 9,3\% del alumnado total, y el curso 1967-68, son 389 representando el 19,8\% (INE 1969). 


\subsection{Comparación y evolución de las matriculadas y las licenciadas.}

La Tabla 1 muestra el número de mujeres que empiezan la carrera de Derecho entre los años 1915 y 1932 y cuantas de ellas se licencian, entendiendo por "licenciada", no la que terminó los estudios sino aquella que solicitó la expedición del título. Pues, así como no existe ningún registro de las personas que finalizaron los estudios, sí lo hay de las personas solicitaron la expedición del título.

Por otro lado, la fecha de expedición del título no necesariamente debe coincidir con la de finalización de los estudios, por lo que puede haber mujeres que hayan empezado y terminado los estudios entre 1927 y 1968 pero que no hayan pagado el título hasta más tarde de 1968 y, por lo tanto, que estén licenciadas, pero no aparezca reflejado en este artículo porque el mismo no comprende el estudio de los años posteriores al 1968.

En base a este extremo, es preciso dejar bien claro que la relación entre matriculadas y licenciadas que se establece en este apartado respecto a los cursos 1915-16 hasta el 1932-33 (Tabla 1) parte de la siguiente presunción: si las estudiantes de este periodo no solicitaron la expedición del título antes del 1968, ya no lo hicieron más tarde.

Observamos que de las 38 mujeres que empezaron la carrera de Derecho en la Facultad entre 1915 y 1932, solo 12 se licenciaron, representando así el 31,5\%, es decir, ni una tercera parte de la cohorte inicial (reg. títulos licenciado 1927-1968).

Por lo que se refiere a los motivos del bajo número de licenciadas, Nash $(1983,16)$ indica que el prototipo de mujer de finales del siglo pasado y hasta bien entrado el siglo XX viene definido por la siguiente atribución: el raciocinio, la lógica, la reflexión, la capacidad de análisis, la creatividad, el rendimiento y la capacidad intelectual predominarían en el hombre, mientras que en la mujer, predominan los sentimientos, el afecto, la sensibilidad, la dulzura, la intuición, la pasividad y la abnegación. De esta manera las características atribuidas a la mujer hacen que se la considere la persona más idónea para ocuparse de la esfera privada, y al hombre de la esfera pública de la política, el derecho y el trabajo.

Asimismo, otras opiniones preveían una extensión de la misión de la mujer más allá del hogar. La visión de la feminista catalana Carmen Karr (1865-1943), directora de la revista Feminal e impulsora de una mejora de la situación de la mujer, preveía la instrucción, formación cultural e incluso profesional de la mujer. Sin embargo, lo que 
constituye el eje principal del pensamiento de Karr es precisamente su interés en facilitar, mediante la cultura y la educación, un mejor cumplimiento de los papeles tradicionales de madre, hija y esposa (Nash, 1983, 17-18).

¿Cómo se explica entonces esta aceptación por parte de la mayoría de las mujeres de una opción que las obligaba a entrar en una situación de inferioridad respecto a su marido? Según Nash $(1983,22)$, los motivos que explican la elección de este modelo de vida son numerosos. Eran determinantes en el amparo de la institución familiar como unidad elemental de la sociedad, la importancia de la doctrina católica y la religión en torno al matrimonio y la familia. También la conveniencia económica, pues el trabajo asalariado femenino era algo transitorio hasta la consecución de un marido y se consideraba el último recurso ante la miseria. De esta manera, la falta de recursos hacía que la mujer considerara el matrimonio como la única manera de asegurar su futuro bienestar económico.

Como se ha comentado anteriormente, aquí hacemos referencia, no al número de mujeres que terminaron los estudios sino al número de mujeres que pagaron el título. Es necesario para el posterior ejercicio del Derecho, la expedición del título. Sin embargo, conociendo el papel de la mujer de la época, es muy probable que la mayoría de las que acababan los estudios, no solicitaran el título porque no tenían la intención de ejercer y que, por tanto, el número de mujeres que acabaron los estudios fuera bastante más elevado que el número de títulos expedidos.

Por otra parte, en cuanto al momento en que las doce licenciadas obtienen el título, siete lo obtienen al cabo de cinco años de haber comenzado, es decir, sin alargar la duración normal de la carrera que, en aquella época, duraba cinco años. Se trataba de:

- Maria de la Asunción Soteras Mauri, licenciada el 1927.

- Maria del Pilar Padrosa Allué, licenciada el 1930.

- Margarita Escoda Colomé, licenciada el 1933.

- Llibertat Gràcia i Teixeria, licenciada el 1933.

- Teresa Argemí Melian, licenciada el 1935.

- Florentina Boadella Clota, licenciada el 1935 
- Maria del Carmen Torres Minguell, licenciada el 1936.

Otras cinco licenciadas solicitan y obtienen el título después de más de cinco años desde el inicio de los estudios; se trata de:

- Maria de las Nieves Miró y Comas, matriculada en 1925, obtiene el título después de 7 años, en 1931.

- Carmen Isern Galcerán, matriculada en 1926, obtiene el título después de 16 años, en 1941.

- Rosa Pujol Burdanova, matriculada en 1930, obtiene el título después de 37 años, en 1967.

- Ana Valls Ventura, matriculada en 1931, obtiene el título tras 11 años, en 1941.

- Maria Rabassa Anguera, matriculada en 1932, obtiene el título después de 10 años, en 1942.

\subsection{Comparación y evolución de las licenciadas y las colegiadas.}

Además de la oferta y la demanda, algunos de los muchos factores que regularon el acceso de la población femenina a la abogacía fueron el carácter masculino que se asignaba a la profesión, el querer alejarlas de los centros formativos que preparaban para su ejercicio o el hecho de que se desarrollara fuera del hogar y en espacios compartidos por hombres (Flecha, 1989).

Para demostrar la situación de desigualdad social y de género que las rodeaba, basta detenerse en algunos hechos concretos que evidencian una manera de actuar que dio prioridad a la división sexual del trabajo asignando y definiendo el doméstico como la contribución específica de las mujeres al crecimiento económico.

De acuerdo con los datos que muestra la Gráfica 2, de las 9 mujeres que se licencian entre los años 1927 y 1936, todas se colegian. Sin embargo, hay que tener presentes dos cuestiones ya comentadas: en primer lugar, es un número muy reducido comparado con el número de alumnos que habían comenzado la carrera hasta 1932, concretamente, 37; $\mathrm{y}$, en segundo lugar, dado que solo han quedado registradas las alumnas que pagaron el título de licenciatura, no podemos saber cuál es la relación real entre las que terminaron los estudios y las colegiadas (ICAB Guías). 
No solo el retraso en la incorporación a los estudios y al ejercicio de la abogacía ha marcado la trayectoria profesional de las abogadas, también la imposibilidad de formar parte de los Cuerpos Jurídicos. Solo pudieron incorporarse en algunos de ellos en los años treinta, durante la II República, pues, desde abril de 1931 hasta mayo de 1933, además de incluir en la Constitución, en el art. 40, que "todos los españoles sin distinción de sexo son admisibles a los empleos y cargos públicos, según su mérito y capacidad", el Gobierno publicó una serie de Decretos que reconocían su derecho a formar parte del Tribunal del Jurado, opositar a Notarías y Registradores de la Propiedad y a ejercer el cargo de Procurador de los Tribunales, pero no el acceso a la Judicatura y a la Fiscalía. Por poco tiempo, pues las Licenciadas en Derecho vieron de nuevo como se les negaban derechos por razón de su sexo cuando estas normativas fueron derogadas por el régimen de Franco al final de la Guerra Civil. Hasta 1966 no pudieron retomar el camino en el acceso gradual a las diferentes profesiones jurídicas (Yanes, 2015).

La Gráfica 2 muestra como de 1940 hasta el 1943, de las 5 licenciadas que había, ninguna se colegió. El número de colegiadas a partir de 1940 en adelante se mantuvo muy bajo respecto al número de licenciadas. Si nos fijamos, hasta el 1955, así como sucedió durante el período anterior al inicio de la Guerra Civil, en ningún caso se incorporaron al colegio más de dos mujeres por año. Así, de las 49 mujeres que se licenciaron entre los años 1940 y 1955, solo 13 se colegiaron, representando el 26,5\%. Se ponen de manifiesto, por tanto, las consecuencias que el franquismo tuvo sobre la mujer.

Finalmente, entre 1956 y 1968, había 166 licenciadas, de las cuales se colegiaron 66, representando el 39,7\%. Es decir, en 12 años, no solo hay un aumento general del número de licenciadas y de colegiadas, sino que el porcentaje que representan las colegiadas sobre las licenciadas aumenta casi en un 2\% respecto a los 28 años anteriores (un $37,9 \%$ de colegiadas sobre las licenciadas). Estos resultados anuncian el cambio que empezó a producirse a partir de 1960 caracterizado por aquella época de desarrollismo en la que la situación social de la mujer comienza a ser percibida de manera diferente a lo que hasta entonces había sido considerada. 


\subsection{Abogadas con y sin ejercicio}

Hasta el inicio de la Guerra Civil española, hay años en los que el número de abogadas en ejercicio supera al de abogadas sin ejercicio y otros años que pasa lo contrario. Vemos que, a lo largo de esos años, el número más elevado de colegiadas en ejercicio se alcanza en 1935-36 con un total de 4, el de colegiadas sin ejercicio en 1933-34 con 4 también y el máximo en general en 1935-36, con 7. En cambio, en el caso de los hombres, el número más elevado, tanto de colegiados en ejercicio, como de colegiados sin ejercicio, como en general, se alcanza en 1935-36, con un total de 960, 739 y 1699 respectivamente.

A partir del año 1939, con el nuevo régimen franquista, el número de colegiadas en ejercicio siempre se mantiene por debajo del de colegiadas sin ejercicio y, antes de entrar en el análisis de las gráficas número 4 y 5, cabe destacar alguna normativa legal en relación al trabajo de la mujer que ponga de manifiesto la mentalidad sobre el papel que estaba llamada a desarrollar en ese momento; papel que la limitaba en el ejercicio de profesiones fuera del hogar y que ayuda a entender el contexto en que se plantea su condición de colegiada en Derecho.

Como dice Flecha $(1989,78)$, la falta de marido y/o la escasez económica es lo que justifica fundamentalmente el trabajo de la mujer. Éste no se contempla como una vía de realización personal y social, lo que se manifiesta en cuestiones como el plus familiar que recibe el marido de la mujer que no trabaja fuera del hogar o la privación del mismo si lo hace, según Orden de 26 de marzo de 1946, el cumplimiento de condiciones (mujer viuda, o soltera, o cabeza de familia, que no pueda hacer frente a sus necesidades por falta de recursos, según Ley de 13 de julio de 1940, sobre Empleados Públicos) para poder presentarse a determinadas oposiciones, los incentivos, o incluso la obligación de abandonar el trabajo cuando accede al matrimonio, o el veto expreso de desempeñar múltiples profesiones y determinados cargos de la Administración del Estado.

En este sentido, podemos observar como desde 1939 hasta 1957, mientras el número de abogadas en ejercicio se mantiene más o menos igual que en el tramo anterior (19261936), el número de abogadas sin ejercicio aumenta. Así, en 1956-57, había 4 abogadas en ejercicio ante 17 sin ejercicio, representando las que ejercían un 19\% del total de las colegiadas. 
La situación empieza a cambiar, al menos formalmente, en 1961, con la aprobación de la Ley 56/1961, de 22 de julio, sobre derechos políticos, profesionales y de trabajo de la mujer que, aún con excepciones, «reconoce a la mujer los mismos derechos que el hombre para todo tipo de actividades políticas, profesionales y de trabajo» (art. 10). La actualización de la normativa respecto al trabajo de la mujer, así como las mejoras en su formación, se hizo imprescindible con el desarrollo económico que se iniciaba en el país, pues éste la iba incorporando de forma progresiva al mundo laboral (Flecha, 1989, $78)$.

Así, observamos que es entre 1960 y 1968 cuando el número total de colegiadas aumentó en mayor medida y, a pesar de que el número de abogadas con ejercicio se mantuvo siempre por debajo del de abogadas sin ejercicio, las dos variables fueron acercándose cada vez más, hasta el punto de que el 1967-68, ejercían 35 abogadas ante 39 que no lo hacían (la diferencia solo era de 4 abogadas), representando las que ejercían el 47,2\% de la totalidad de colegiadas.

\section{Conclusiones}

La presencia de la mujer en la Facultad de Derecho fue ínfima durante las primeras décadas del siglo XX. Podemos confirmar que la primera mujer que se matriculó en la Facultad de Derecho de la Universidad de Barcelona a partir de 1910 lo hizo en 1916 con el nombre de María del Amparo Roig García. Venía de traslado de Madrid y solo se matriculó de una asignatura: Derecho Administrativo. En 1930, veinte años después de haberse regulado la admisión de las mujeres en la universidad en las mismas condiciones que el hombre, solo eran nueve las mujeres matriculadas y representaban el $0,8 \%$ de la totalidad del alumnado.

La inmensa mayoría de las estudiantes de Derecho no acababan los estudios o bien, a pesar de terminarlos, no solicitaban la expedición del título de licenciado necesario para el posterior ejercicio, pues se potenció el discurso de la mujer profesional, educada, que podía entrar en la esfera pública, mientras fuera soltera y no tuviera responsabilidades familiares domésticas.

De las 38 mujeres que empezaron la carrera entre 1915 y 1932, solo 12 se licenciaron, representado el 31,5\%, ni una tercera parte. A pesar de ser siempre minoritaria, la matrícula femenina fue en aumento desde sus inicios, sobre todo con la llegada de la II 
República, pues en 1932, eran 55 las mujeres matriculadas representando el 4,3\% de la totalidad del alumnado. Tras la Guerra Civil, se produjo la reincorporación de algunas mujeres a la Facultad, pero no fue hasta el 1953 que el número de alumnas volvió a acercarse al 5\% del alumnado.

La respuesta represora del franquismo hacia las mujeres fue muy intensa y se produjo un proceso de aculturación en unos valores ultraconservadores que volvieron a anular oficialmente al sujeto femenino de la esfera pública. Se potenciaron unos roles de género que instrumentalizaron a la mujer, y que se legitimaban a partir de un cuerpo legislativo de discriminación legal y de subordinación social.

Así, a pesar de la reincorporación y el aumento de la matrícula femenina en la Facultad, su número no se correspondía con el de licenciadas y aún menos con el de colegiadas. A diferencia de lo que sucedía en el caso de los hombres, el número de abogadas sin ejercicio superó en todo momento el número de abogadas con ejercicio. Es a partir de los años 60, con la época del desarrollismo, cuando el número de matriculadas, licenciadas, colegiadas y su ejercicio profesional empezó a acercarse en mayor medida.

Es sorprendente y queda patente que, a partir del 1910, el proceso de incorporación de las mujeres a la Facultad parece irreversible. Los años de dictadura son desagradables para las mujeres y sus reivindicaciones, pero, incluso durante esta etapa, su incremento porcentual sobre el conjunto del alumnado universitario no dejó de crecer.

En todo caso, el tema de la incorporación de la mujer en el mundo del Derecho es tan amplio que son muchas las cuestiones que quedan por estudiar. Así, pienso que sería interesante realizar en un futuro otros trabajos consistentes en, por ejemplo, prolongar la presente investigación hasta la actualidad, ver a partir de los expedientes académicos cuántas y cuáles fueron las mujeres que efectivamente terminaron los estudios, ver cuál era su procedencia o incluso efectuar un estudio de carácter cualitativo centrado en la vida de algunas de las pioneras. 


\section{Referencias bibliográficas}

ACKELSBERG, M. A. (2017). Mujeres Libres: el anarquismo y la lucha por la emancipación de las mujeres. Pròleg de Cris Tejada i Lorena Martín. Barcelona: Virus.

ANDREU, J., LÓPEZ, M. y VERNET, M.T. (2013). El archivo histórico de la Universitat de Barcelona: recurso para la investigación en historia de las universidades. CIAN-Revista de Historia de las Universidades, 16/2 (2013), 123-139

DEL AMO DEL AMO, M. C. (2009). La educación de las mujeres en España: de la “amiga" a la Universidad. CEE Participación educativa, vol. 11, no 1, p. 8-22.

FLECHA GARCÍA, C. (1989) Algunos aspectos sobre la mujer en la política educativa durante el régimen de Franco. Historia de la Educación, 8, 77-98.

FOGUET I BOREU, F. (2018). Maria Aurèlia Capmany, escriptora compromesa (19631977). Barcelona: Abadia de Montserrat.

FORASTER SIDRACH, P. (2015). El Moviment de les dones a Catalunya des de finals del segle XIX fins a mitjans del segle XX: dues fractures del moviment feminista, 19311936. 1-57.

FULLOLA PERICOT, M. (2014). Breu història de la Universitat de Barcelona. Barcelona: Publicacions i Edicions de la Universitat de Barcelona.

GARCÍA LASTRA, M. (2010). La voz de las mujeres en la universidad. Revista de la Asociación de Sociología de la Educación (RASE), vol. 3, no 3, p. 357-368.

GÓMEZ BLESA, M. (2009). Modernas y vanguardistas: mujer y democracia en la II República. Madrid: Laberinto.

ITATÍ PALERMO, A. (2006). El acceso de las mujeres a la educación universitaria. Revista argentina de sociología, vol. 4, no 7.

MORENO CULLELL, V. (2010). El llarg camí cap a la igualtat de la dona: el segle XX com a segle de les dones. Blog Sàpiens.

NASH, M. (1983). Mujer, familia y trabajo en España (1875-1936), Anthropos Editorial, 1983. 
YANES, J. (2015). Estudio histórico-jurídico del acceso de la mujer a la abogacía en España (tesis doctoral). Universidad de las Palmas de Gran Canaria.

\section{Fuentes archivísticas}

[Memòria] Archivo de la Universidad de Barcelona (2014), Memòria de la llum: "femina perfundet omnia luce" [exposición en línea].

Archivo de la Universidad de Barcelona, Libros de registro de matrículas en la Facultad de Derecho de la UB (1910-1968)

Archivo de la Universidad de Barcelona, Libros de registro de los títulos de licenciado en Derecho por la UB (1910-1947 y 1957-1969)

Archivo de la Universidad de Barcelona, Relación de títulos de licenciado que se han pagado de las diferentes facultades de la UB (1947-1957)

ICAB, Guías Judiciales del ICAB (1927-1968) 